$39^{\text {th }}$ AIAA/ASME/SAE/ASEE

AIAA-2003-5097

Joint Propulsion Conference and Exhibit

Von Braun Center, Huntsville, Alabama

July 20-23, 2003

\title{
ANALYSIS OF PROSEDS TEST OF BARE-TETHER COLLECTION
}

J.R. Sanmartín

Universidad Politécnica de Madrid

Madrid, Spain

Email: jrs@faia.upm.es

E.C. Lorenzini
Harvard-Smithsonian
Center for Astrophysics
Cambridge, MA 02138
Email: elorenzini@cta.harvard.edu

R.D. Estes

OnScreen Science, Inc.

Somerville, MA 02140

Email: estes@onscreen-sci.com
M. Charro

Universidad Politécnica de Madrid

Madrid, Spain

M.L. Cosmo

Harvard-Smithsonian

Center for Astrophysics

Cambridge, MA 02138

Email: mcosmo(acfa, harvard.edu

\section{ABSTRACT*}

NASA's tether experiment ProSEDS will be placed in orbit on board a Delta-II rocket to test bare-tether electron collection, deorbiting of the rocket second stage, and the system dynamic stability. ProSEDS performance will vary because ambient conditions change along the orbit and tether-circuit bulk elements at the cathodic end follow the step-by-step sequence for the current cycles of operating modes (open-circuit, shunt and resistor modes for primary cycles; shunt and battery modes for secondary cycles). In this work we discuss expected ProSEDS values of the ratio $L_{l} / L^{*}$, which jointly with cathodic bulk elements determines bias and current tether profiles; $L_{t}$ is tether length, and $L^{*}$ (changing with tether temperature and ionospheric plasma density and magnetic field) is a characteristic length gauging ohmic versus baretether collection impedances. We discuss how to test bare-tether electron collection during primary cycles, using probe measurements of plasma density, measurements of cathodic current in resistor and shunt modes, and an estimate of tether temperature based on ProSEDS orbital position at the particular cycle concerned. We discuss how a temperature missestimate might occasionally affect the test of bare-tether collection, and how introducing the battery mode in some primary

*Copyright (C) 2003 by the American Institute of Aeronautics and Astronautics, Inc. All rights reserved. cycles, for an additional current measurement, could obviate the need of a temperature estimate. We also show how to test bare-tether collection by estimating orbit-decay rate from measurements of cathodic current for the shunt and battery modes of secondary cycles.

\section{INTRODUCTION}

NASA's Propulsive Small Expendable Deployer System (ProSEDS) will fly as secondary payload on a Delta II rocket to demonstrate propulsion by a bare electrodynamic tether'. The launch date of ProSEDS had been scheduled for early 2003, with deployment upwards from the second stage of the rocket in a $360 \mathrm{~km}$ nearly circular orbit at an inclination of 36 . The geomagnetic field would both induce an electric field on the orbiting tether and exert a dragging force on the resulting current, strongly increasing the decay rate of the Delta stage. ProSEDS launch should now take place in February 2004.

The ProSEDS tether is about $15 \mathrm{~km}$ long. The $10 \mathrm{~km}(10,132 \mathrm{~m})$ segment first deployed is a nonconductive Dyneema tape of average crosssection $1.2 \mathrm{~mm} \times 0.2 \mathrm{~mm}$, with a $20 \mathrm{~m}$ leader made of Kevlar, total weight being about $1.5 \mathrm{~kg}$. Following the tape is a $5-\mathrm{km}(5,080 \mathrm{~m})$ set of aluminum wires of $1.2-\mathrm{mm}$ outer diameter, left bare except for its last $215 \mathrm{~m}$ which are insulated and overbraided with Kevlar; the total weight of the conductive segment is $10 \mathrm{~kg}$. The bare segment has 
a C-COR coating that protects aluminum against atomic oxygen and modifies optical properties for passive control of wire temperature, to keep resistivity low and to preserve mechanical behavior. The Delta second stage has a mass of $994 \mathrm{~kg}$, while the body attached to the other end of the tether for deployment and stability purposes has a $21.4 \mathrm{~kg}$ mass.

Electrons are collected over some upper portion of the bare wire that will become positively biased with respect to the surrounding plasma. The full electron current collected is reemitted into the ionosphere by an active hollow-cathode device at the Delta stage. The Hollow Cathode will keep the platform at less than $30 \mathrm{~V}$ of the local plasma potential. Estimated average currents, depending on ambient conditions, are $1.3-1.4 \mathrm{~A}$ with about 3.5 A peak values, corresponding drag being $0.15 \mathrm{~N}$ and $0.4 \mathrm{~N}$ respectively. Part of the energy taken from the orbital motion will be used to recharge batteries that might allow for extended measurements, using, in particular, Langmuir probes and a differential-ion-flux probe. ProSEDS current is run under two different cycles of operation. An orbit-decay rate of about $11 \mathrm{~km} /$ day is expected for the first 5 orbits, covering the first type of cycle, and then increasing for the remaining orbits.

\section{PROSEDS CURRENT COLLECTION AND PROFILE}

Parameters characterizing an orbiting tether and the magnetized ionospheric plasma at LEO determine three dimensionless numbers that are all small for ProSEDS: These numbers are the ratios Debye length/electron thermal-gyroradius, tether radius/Debye length and ion ram energy/ $e \Delta V$. The electron thermal gyroradius is about 3-10 times greater than the Debye length, itself typically 10 times greater than the radius of the ProSEDS tether. The ion ram energy is about $5 \mathrm{eV}$ for oxygen, whereas $\Delta V$, which is the tether bias relative to the ionosphere, is of order of the full induced electromotive force, $\varepsilon_{\text {ind }}=E_{m} L_{t}$, and is much greater than $5 \mathrm{~V}$; here $L_{t} \approx 5 \mathrm{~km}$ is the conductive length of tether and $E_{m}$ is the motional electric field (product of orbiting speed and geomagnetic-field component perpendicular to the orbital plane). Simulations indicate that the tether stays straight and close to vertical during the full first day in orbit, particularly over the early five orbits. Figure 1 shows expected values for $\varepsilon_{\text {ind }}$ from a particular numerical simulation of the ProSEDS orbital evolution ${ }^{2}$.

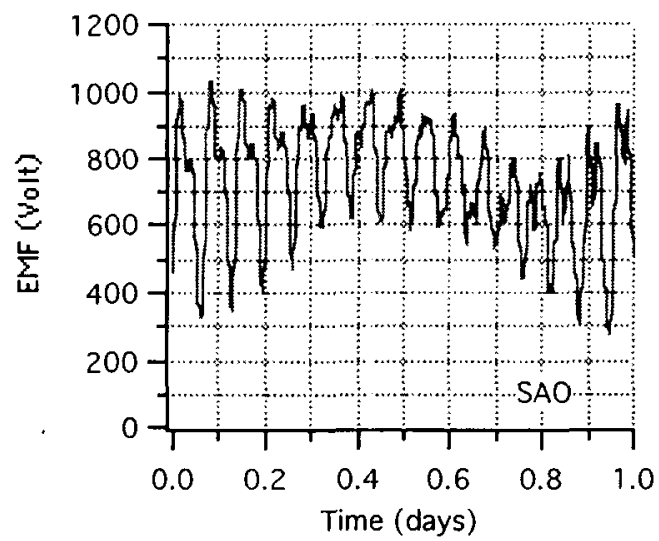

Fig. 1 Electromotive force from simulation results for solar radio flux F10.7 = 140 .

Current collection by unit length of ProSEDS bare-tether is then presumed to follow the orbitalmotion-limited (OML) law, which is just proportional to $N_{x} p \sqrt{ } \Delta V$, where $p$ is perimeter of tether cross-section and $N_{\infty}$ is ambient plasma density $^{3}$. Figure 2 shows $N_{\infty}$, for the same simulations of Fig. 1. ProSEDS will particularly serve to verify in space that ram effects on ions due to the large ion Mach number, or even geomagnetic effects on electrons, do not invalidate the OML law.

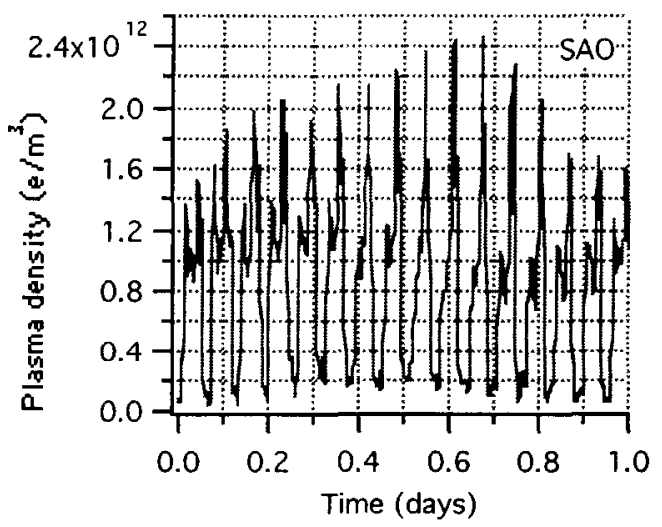

Fig. 2 Plasma density from Fig. 1 simulation.

The actual variation along tether length of bias $\Delta V$ and current $I$ flowing in the tether are determined by a characteristic length $L^{*}$ that gauges ohmic versus OML-collection impedance (see next section), and is defined by ${ }^{4}$

$$
L^{*} \times e N_{\infty} \times \frac{p}{\pi} \sqrt{\frac{2 e E_{m} L^{*}}{m_{e}}}=\frac{3}{4} \sigma A_{c} E_{m}=\frac{3}{4} \frac{\varepsilon_{\text {ind }}}{R_{t}}
$$


where $R$, is tether resistance. The conductive segment of ProSEDS has perimeter $p=\pi \times 1.2$ $\mathrm{mm}$ and is made of aluminum strands; the effective conductive cross-section area $A_{r}$ results in a resistance $R_{t} \equiv L_{\mathrm{t}} / \sigma A_{c}=250 \Omega$ at $20 \mathrm{C},(\sigma \equiv$ Al conductivity).

A fundamental dimensionless parameter for bare-tether performance, $L_{t} / L^{*}$, is given by the expression

$$
\frac{L_{t}}{L^{*}} \equiv l_{t}=4.17 \times\left(\frac{500 \mathrm{~V}}{\varepsilon_{\text {ind }}}\right)^{1 / 3} \times\left(\frac{R_{t}}{250 \Omega} \times \frac{N_{\infty}}{10^{12} m^{-3}}\right)^{2 / 3}
$$

for the ProSEDS tether. Figures 3 and 4 show $R_{t}$ and $l$, for the same simulations of Fig. 1 .

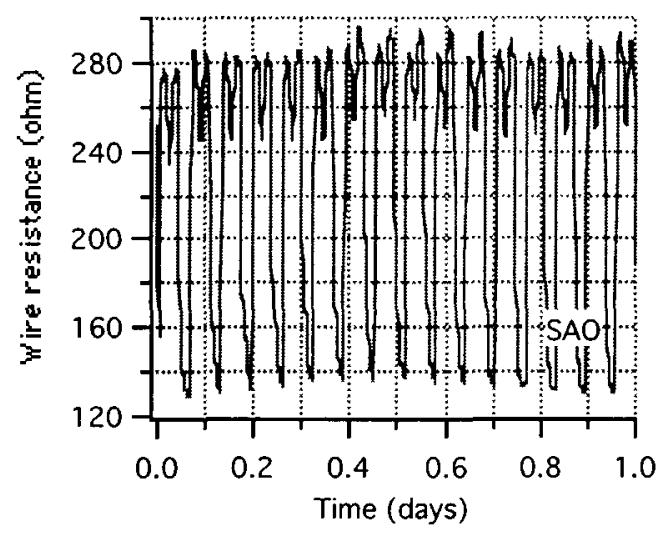

Fig. 3 Tether resistance from Fig. 1 simulation.

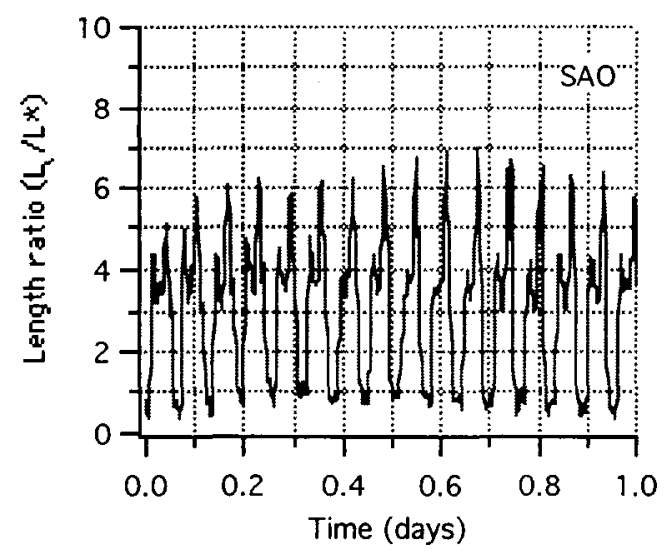

Fig. 4 Length ratio $l_{s}$ as given in Eq. (1) and derived from the results of Figs. 1-3.

\section{CURRENT AND BIAS AT CATHODIC END OF TETHER}

The ProSEDS current is run under two different operating cycles. Primary cycles with period $60 \mathrm{~s}$ (Fig.5) start at Delta Time $=12,666$ seconds and run for 5 orbits. The tether is in open circuit mode (Hollow Cathode disconnected from tether, making it to float electrically) for the first 35 $\mathrm{s}$, with the $\mathrm{HC}$ idle during $30 \mathrm{~s}$ and on during the remaining $5 \mathrm{~s}$. For the rest of each cycle the tether is connected to the $\mathrm{HC}$, running in a resistor mode (with a $Z_{\Omega}=2.32 \mathrm{k} \Omega$ load in the circuit) for 5 seconds, and in shunt mode (tether current shorted to the $\mathrm{HC}$ ) in the final $20 \mathrm{~s}$. For the currents of interest the Hollow Cathode may be characterized by a negative resistance $r_{H C}=-4 \Omega$ and a limit potential drop at low current $\Phi_{H C}=40 \mathrm{~V}$.

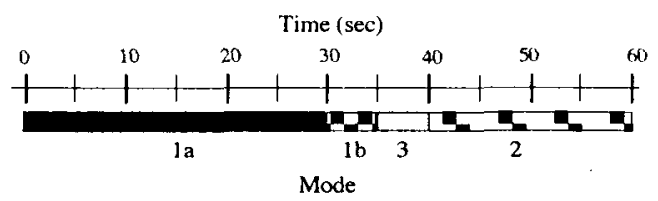

Fig. 5 First operating cycle: modes open circuit (la) plasma contactor idle; (1b) contactor on; shunt (2); resistor (3).

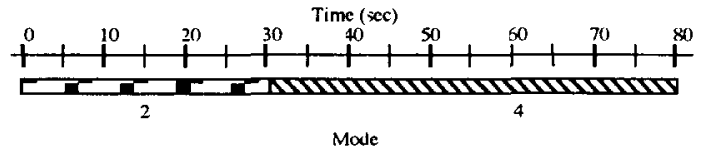

Fig. 6 Second operating cycle: modes shunt (2) and battery charge (4).

The secondary operating cycle that is activated after 5 orbits has a period of 80 seconds (Fig. 6). The tether runs in the shunt mode for the first $30 \mathrm{~s}$ and in a battery mode for the rest of the cycle. The battery has negligible internal resistance and an electromotive force $\varepsilon_{\text {bat }}=120 \mathrm{~V}$. From Figs. 1 and 3 we have $\Phi_{H C} \ll \varepsilon_{\text {bat }} \ll \varepsilon_{\text {ind }}$ and $\left|r_{H C}\right| \ll R_{t} \ll$ $Z_{\Omega}$.

Whichever bulk elements at the cathodic end $C$ of the tether, that is, whichever the mode, the bias $\Delta V$ relative to the plasma varies along tether length due to both ohmic and induced electric-field drops,

$$
\frac{d \Delta V}{d s}=\frac{I(s)}{\sigma A_{c}}-E_{m}
$$


with $s$ running from anodic top $A$ to bottom $C$ (Fig. 7). Over a segment $A B$ with $\Delta V>0$, electrons are collected at the rate given by the OML law,

$$
\frac{d I}{d s}=e N_{\infty} \times \frac{p}{\pi} \times \sqrt{\frac{2 e \Delta V(s)}{m_{e}}}
$$

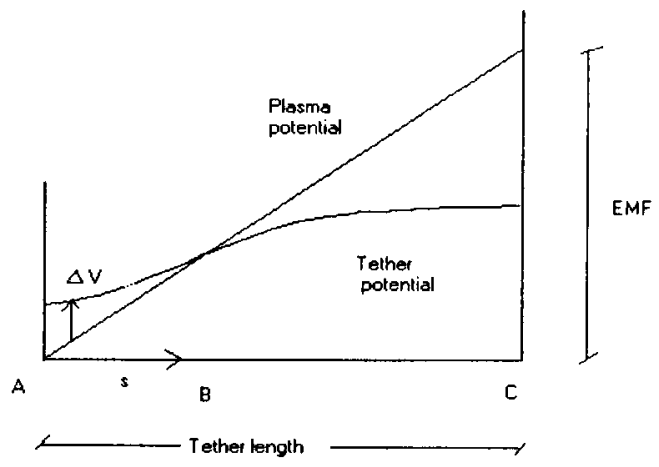

Fig. 7 Voltage diagram. A bulk element at cathodic end $C$ relates current and bias there.

Equations (2), (3) are readily integrated, with results conveniently displayed in dimensionless variables $^{4}$

$$
\begin{aligned}
& i \equiv I / \sigma A_{c} E_{m} \quad(<1), \quad \varphi \equiv \Delta V / E_{m} L^{*}, \\
& l \equiv s / L^{*} .
\end{aligned}
$$

One finds $\varphi^{3 / 2}+2 i-i^{2}=$ constant and (using conditions $i_{A}=\varphi_{B}=0$ )

$$
\varphi_{A}^{3 / 2}=2 i_{B}-i_{B}^{2},
$$

$\frac{\text { length } A B}{L^{*}} \equiv l_{B}\left(i_{B}\right)=\int_{0}^{\varphi_{A}^{\left(i_{B}\right)}} \frac{d \varphi}{\sqrt{\left(1-i_{B}\right)^{2}+\varphi^{3 / 2}}}$

with $l_{B} \approx\left(2 i_{B}\right)^{2 / 3}$ at small $i_{B}$ and $l_{B}(1)=4$.

lons are collected over the segment $B C$ with $\Delta V<0$ according to the OML law too, at a rate $\mu \equiv \sqrt{ } m_{e} / m_{i}$ times smaller than electrons,

$$
\frac{d I}{d s}=-\mu \times e N_{\infty} \times \frac{p}{\pi} \sqrt{\frac{2 e|\Delta V|}{m_{e}}}
$$

From Eqs. (2) and (3') one readily obtains $\mu|\varphi|^{3 / 2}+$ $2 i-i^{2}=$ constant and, using condition $\varphi_{B}=0$,

$$
\mu\left|\varphi_{C}\right|^{3 / 2}+2 i_{C}-i_{C}^{2}=2 i_{B}-i_{B}^{2}
$$

$$
\begin{aligned}
& l_{t}-l_{B}\left(i_{B}\right)=\int_{0}^{\left|\varphi_{C}\right|} \frac{d|\varphi|}{1-i} \equiv \\
& \left|\varphi_{C}\right| \times \int_{0}^{1} \frac{d x}{\sqrt{\left(1-i_{C}\right)^{2}-\mu\left|\varphi_{C}\right|^{3 / 2}\left(1-x^{3 / 2}\right)}}
\end{aligned}
$$

With Eqs. $(5,6)$ giving $l_{B}\left(i_{B}\right)$, Eqs. (7) and (8) determine a relation between dimensionless current $i_{C}$ and bias $\left|\varphi_{C}\right|$ at tether end $C$, involving parameters $l_{t}$ and $\mu\left|\varphi_{c}\right|^{3 / 2}$. For $\mu \approx 1 / 170$ (oxygen ions) and values of $l$, as shown in Fig. 4 , $\mu l_{t}^{3 / 2}$ is small. Since we clearly have $\left|\varphi_{d}\right|<l$, we also have $\mu\left|\varphi_{c}\right|^{3 / 2}$ small. One easily shows that our having ignored the insulation over a short segment of tether above bottom $C$ results in corrections that are of order higher than $\mu\left|\varphi_{C}\right|^{3 / 2}$ and $\mu^{2 / 3}$.

A second equation for $i_{C}$ and $\left|\varphi_{C}\right|$ is provided by the circuit relation for the bulk element at $C$, which is different for each different mode. For the open circuit mode we just have

$$
i_{C}=0 .
$$

For the resistor mode we have

$$
\begin{aligned}
& \left|\varphi_{C}\right|=\frac{\Phi_{H C}+\left(Z_{\Omega}+r_{H C}\right) I_{C}}{E_{m}{ }^{*}}= \\
& l_{t} \times\left[\frac{\Phi_{H C}}{\varepsilon_{\text {ind }}}+\frac{Z_{\Omega}+r_{H C}}{R_{t}} i_{C}\right]
\end{aligned}
$$
have

For the shunt and battery modes we similarly have

$$
\frac{\left|\varphi_{C}\right|}{l_{t}}=\frac{\Phi_{H C}}{\varepsilon_{\text {ind }}}+\frac{r_{H C}}{R_{t}} i_{C}
$$

$$
\frac{\left|\varphi_{C}\right|}{l_{t}}=\frac{\varepsilon_{b a t}+\Phi_{H C}+r_{H C} I_{C}}{\varepsilon_{i n d}}
$$$$
=\frac{4 \Phi_{H C}}{\varepsilon_{i n d}}+\frac{r_{H C}}{R_{t}} i_{C}
$$

respectively. 
Equations (5-8) together with the Eq.(9) corresponding to each mode show how current $i_{C}$ and bias $\left|\varphi_{C}\right|$ at tether end $C$ may be predicted once the bulk element at $C$ is defined, if OML currentcollection is valid (and ambient conditions and tether parameters are known). Then one just writes $I_{C}=i_{C} \times \varepsilon_{\text {ind }} / R_{\imath}$ and $\left|\Delta V_{C}\right|=\left|\varphi_{C}\right| \times E_{m} L^{*}$ for dimensional current and bias.

\section{TESTS OF OML CURRENT COLLECTION FROM PRIMARY-CYCLE DATA}

There are about 450 primary cycles, each one providing, in principle, a test of the OML law. Equation (1) for the dimensionless length $l_{r}$ can be rewritten as an equation for the plasma density,

$\frac{N_{\infty}}{10^{12} m^{-3}} \approx \frac{250 \Omega}{R_{t}} \times\left(\frac{\varepsilon_{\text {ind }}}{500 \mathrm{~V}}\right)^{1 / 2} \times\left(\frac{l_{t}}{4.17}\right)^{3 / 2}$

Measurements of $\Delta V_{C}$ during the open-circuit mode, and $I_{C}$ during the resistor and shunt modes of a primary cycle might in principle determine all three quantities $\varepsilon_{\text {ind }}, R_{t}$, and $l_{s}$, yielding $N_{x}$ in (1'). Direct measurement of plasma density, which will be carried out during the first 5 orbits using a Langmuir probe, will yield an independent value for $N_{\infty}$, to be compared with $\left(1^{\prime}\right)$.

Note that, as shown in Figs. 1-4, variations of $\varepsilon_{\text {ind }}, N_{\infty}, R_{t}$, and $l_{t}$ are expected to cover a large parametric domain. This will result in very broad testing. On the other hand we may safely assume that tether temperature (and thus tether resistance $R_{t}$ ), and both plasma density $N_{\infty}$ and motional electric field $E_{m}$ (leading to the length ratio $l_{t}$ ) change little during a cycle $(60 \mathrm{~s}$ in time or about $450 \mathrm{~km}$ in distance), except possibly when crossing the terminator.

It turns out, however, that both $\Delta V_{C}$ in the open-circuit mode and $I_{C}$ in the resistor mode basically determine the induced electromotive force $\varepsilon_{\text {ind. }}$. From Eqs. $(7,8)$ and $(9 a)$ or $(9 b)$ one would approximately find $\varepsilon_{\text {ind }} \approx\left|\Delta V_{C}\right|$ (open circuit) or $\varepsilon_{\text {ind }} \approx Z_{\Omega} \times I_{C}$ (resistor). This has a positive side, because measuring $\Delta V_{C}$ in the open-circuit mode is problematic. (Intended direct measurements of the motional electric field using magnetometers present uncertainties too.) On the other hand, it then becomes impossible to obtain both $R_{t}$ and $l_{t}$, for use in Eq.(1'), from the single remaining measurement of $I_{C}$ in the shunt mode.

Fortunately, tether temperature is basically governed by solar irradiation, and is weakly affected by Joule heating, or by heating arising from the impact of the attracted particles. As a result, there might be no need for measuring tether resistance, which might be reasonably predicted from the ProSEDS orbital position at the particular cycle considered, independently from detailed jonospheric conditions. Ignoring then the open circuit mode and assuming $R_{t}$ known, one uses currents $I_{C}^{\text {res }}$ and $I_{C}{ }^{\text {sh }}$ to determine $l_{t}$ and $\varepsilon_{\text {ind }}$.

The induced electromotive force is obtained from the resistor current measurement. Since $Z_{\Omega} /$ $R$, is large, we have $i_{C}$ small in Eq.(9b), implying small $i_{B}$, and thus $\left|\varphi_{C}\right|$ close to $l_{1}$ in Eq.(8). From Eqs.(8) and (9b), with $\left|\varphi_{C}\right| \approx l_{t}$ and $i_{C} \approx R_{t} / Z_{\Omega}$, one can then find an explicit expression for $\varepsilon_{\text {ind }}$,

$$
\begin{aligned}
& \varepsilon_{\text {ind }}=\left[Z_{\Omega} I_{C}^{r e s}+\Phi_{H C}+R_{t} I_{C}^{r e s}\right] \times \\
& {\left[1+0.3 \mu l_{t}^{3 / 2}+\left(\mu+\frac{2}{l_{t}^{3 / 2}} \times \frac{R_{t}}{Z_{\Omega}}\right)^{2 / 3}\right]}
\end{aligned}
$$

For $l_{r}$ moderately large at most $\left(l_{t}\right.$ less than about 6 in Fig. 4), one can verify in Eqs. (8) and (9c) for the shunt mode, with $\left|\varphi_{C}\right| l$, clearly small, that current and bias at $C$ satisfy condition $\left(1-i_{C}\right)^{2}=$ $\left(1-i_{B}\right)^{2} \gg \mu\left|\varphi_{C}\right|^{3 / 2}$. Then, one can carry out the integral in Eq.(8) approximately. Also, taking $i_{B}$ from Eq.(7) simplified to the form $i_{B}=i_{C}+\mu$ $\left|\varphi_{C}\right|^{3 / 2} / 2\left(1-i_{C}\right)$, one may write $l_{B}\left(i_{B}\right)$ in terms of $i_{C}$ and $\varphi_{C}$,

$$
l_{B}\left(i_{B}\right) \approx l_{B}\left(i_{C}\right)+\frac{\mu\left|\varphi_{C}\right|^{3 / 2}}{2\left(1-i_{C}\right)} \times \frac{d l_{B}}{d i_{C}}
$$

Eq.(8) finally yielding an implicit equation for $l_{t}$,

$$
\begin{aligned}
& l_{t}=l_{B}\left(i_{C}\right) \times\left[1+\frac{\mu\left|\varphi_{C}\right|^{3 / 2}}{2\left(1-i_{C}\right)} \frac{d \ln l_{B}\left(i_{C}\right)}{d i_{C}}\right]+ \\
& \frac{\left|\varphi_{C}\right|}{1-i_{C}} \times\left[1+\frac{0.3 \mu\left|\varphi_{C}\right|^{3 / 2}}{\left(1-i_{C}\right)^{2}}\right]
\end{aligned}
$$

In Eq. (11), $l_{B}\left(i_{C}\right)$ is obtained by setting $i_{B} \rightarrow$ $i_{C}$ in (5) and (6), while $\varphi_{C}$ is given by Eq.(9c) and $i_{c}$ results from reverting to dimensional current, 


$$
\begin{aligned}
\left|\varphi_{C}\right| & \equiv \frac{\Phi_{H C}+r_{H C} I_{C}^{s h}}{\varepsilon_{\text {ind }}} l_{t}, \\
i_{C} & \equiv \frac{R_{t} I_{C} s h}{\varepsilon_{\text {ind }}}
\end{aligned}
$$

with $\varepsilon_{\text {ind }}$ taken from (10).

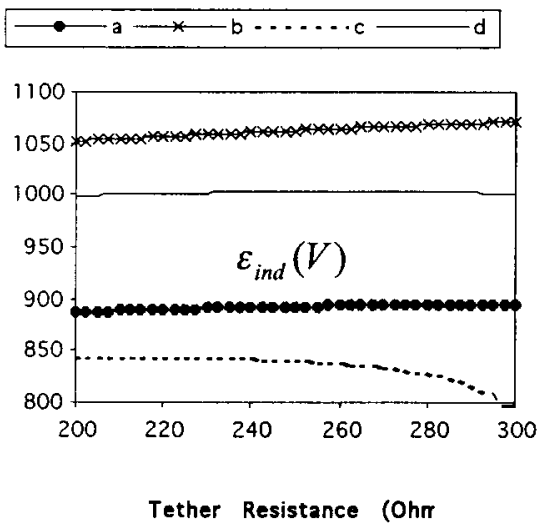

Fig. 8 Induced EMF, as determined by Eqs. (1') and (10)-(12), versus estimated tether resistance. Shunt-mode current was given values $2 \mathrm{Amp}$ for cases $a$ and $b$ and $2.5 \mathrm{Amp}$ for $c$ and $d$; resistor-mode current was $0.26 \mathrm{Amp}$ for cases $a$ and $c$ and $0.3 \mathrm{Amp}$ for $b$ and $d$.
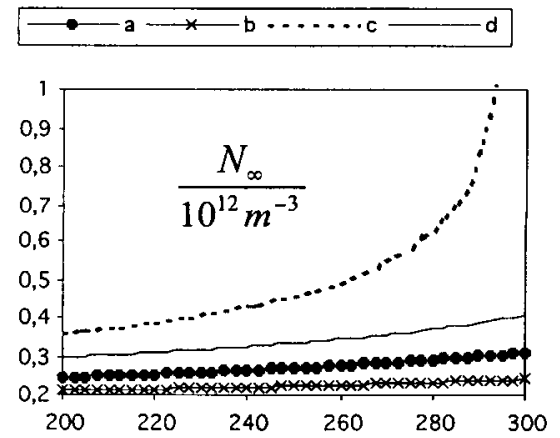

Tether Resistance (Ohr

Fig. 9 Plasma density versus tether resistance; currents as in Fig. 8

For given values of currents $I_{C}^{\text {res }}$ and $I_{C}^{\text {sh }}$ and an estimated value of $R_{t}$ one could thus determine $\varepsilon_{\text {ind }}, l_{\text {t }}$ and finally $N_{\infty}$. Figures 8 and 9 show $\varepsilon_{\text {ind }}$ and $N_{\infty}$ versus $R_{t}$ for particular values of $I_{C}^{\text {res }}$ and $l_{C}^{\text {sh }}$ within expected ranges. In general, an indeterminacy in $R_{l}$ leads to a weaker indeterminacy in $N_{x}$. Case $c$ ), which shows plasma density strongly dependent on tether resistance at its range end, corresponds to values $i_{C}{ }^{\text {sh }}$ very close to unity, for which condition (1$\left.i_{C}\right)^{2} \gg \mu\left|\varphi_{C}\right|^{3 / 2}$ is actually invalid. This suggests, however, that there may be conditions where the OML test will fail.

A way out of this problem would be to use the battery mode in some primary cycles to obviate the need for estimating $R_{r}$. As seen in Eqs. $(9 \mathrm{c}, \mathrm{d})$, equations for the battery mode only differ from those of the shunt mode in the change $\Phi_{H C} \rightarrow 4 \Phi_{H C}$. The approximations leading to (11) still apply $\left(\left|\varphi_{C}\right|\right.$ is now greater but this requires a greater $i_{C}$ too). To explicitly show here the scheme of solution we take the set of equations in their lowest approximation, writing

$$
\begin{aligned}
& \varepsilon_{\text {ind }} \approx Z_{\Omega} I_{C}^{\text {res }} \\
& l_{t} \approx l_{B}\left(i_{C}\right)+\left|\varphi_{C}\right| /\left(1-i_{C}\right)
\end{aligned}
$$

(for shunt and battery modes).

$$
\begin{aligned}
& \left|\varphi_{C}{ }^{s h}\right| \approx l_{r} \times \Phi_{H C} / \varepsilon_{\text {ind }}, \\
& \left|\varphi_{C}{ }^{\text {but }}\right| \approx l_{t} \times 4 \Phi_{H C} / \varepsilon_{\text {ind }} .
\end{aligned}
$$

We then find $i_{C}^{\text {bat }}\left(I_{C}^{\text {sh }} / I_{C}^{\text {bat }}, \quad \Phi_{H C} / Z_{\Omega} I_{C}^{r e s}\right)$ from the equations

$$
\begin{gathered}
l_{B}\left(i_{C}{ }^{b a t}\right) \times\left[1-\frac{\Phi_{H C} / Z_{\Omega} I_{C}^{r e s}}{1-i_{C}{ }^{s h}}\right]= \\
l_{B}\left(i_{C}{ }^{s h}\right) \times\left[1-\frac{4 \Phi_{H C} / Z_{\Omega} I_{C}^{r e s}}{1-i_{C}{ }^{b a t}}\right] \\
i_{C}^{s h} / i_{C}^{b a t}=I_{C}^{s h} / I_{C}^{b a t} .
\end{gathered}
$$

We would finally find $N_{\infty}$ from Eq.(10') together with the equations

$$
R_{t} \approx i_{C}^{b a t} \times \frac{Z_{\Omega} I_{C}^{r e s}}{I_{C}^{b a t}},
$$

$$
\frac{1}{l_{t}}=\frac{1}{l_{B}\left(i_{C}^{b a t}\right)} \times\left[1-\frac{4 \Phi_{H C} / Z_{\Omega} I_{C}^{r e s}}{1-i_{C}^{b a t}}\right]
$$

6 


\section{TEST OF OML CURRENT COLLECTION FROM PROSEDS SECONDARY-CYCLE DATA}

No density measurements will be carried out during secondary cycles. Nonetheless, such cycles, which are most effective in dragging the tether and cover most of the decay time from orbit, can provide a second test of OML collection. Actual measurements of orbital decay, which is dependent on the magnetic-drag power, would be compared to predictions based on the OML law and on current measurements during the shunt and battery modes of each secondary cycle, together with an estimate of $R_{t}$. Differently from the primary-cycle tests, this is an overall test resulting from integrating the drag power over many cycles.

At any given time, magnetic power is proportional to the average tether current,

$$
\begin{aligned}
& W=\text { Orbital Speed } \times \text { Drag }=\varepsilon_{\text {ind }} \tilde{I}, \\
& \tilde{I} \equiv \int_{0}^{L_{t}} I(s) d s / L_{t} .
\end{aligned}
$$

From Eq. (2) one readily finds $\tilde{I} / \sigma A_{C}=E_{m}+$ $\left(\Delta V_{C}-\Delta V_{A}\right) / L_{t}$, leading to

$$
W=\frac{\varepsilon_{\text {ind }}^{2}}{R_{t}} \times\left(1-\frac{\left|\varphi_{C}\right|+\varphi_{A}}{l_{t}}\right) .
$$

The average drag-power over a full secondary cycle would be

$$
W_{a v}(\sec \text { ondary cycle })=\frac{3}{8} W_{s h}+\frac{5}{8} W_{b a t} .
$$

To a first approximation we take $\left|\varphi_{C}{ }^{s h}\right| / l_{t}$, and $\left|\varphi_{C}{ }^{\text {bat }}\right| / l_{t}$ from Eqs.(13a, b) and set $i_{B} \approx i_{C}$ in Eq. (5) to obtain

$$
\begin{aligned}
& W_{a v}(\text { secondary cycle }) \approx \frac{\varepsilon_{\text {ind }}^{2}}{R_{t}}\left[1-\frac{23}{8} \frac{\Phi_{H C}}{\varepsilon_{\text {ind }}}\right]- \\
& \frac{\varepsilon_{\text {ind }}{ }^{2}}{R_{t}}\left[\frac{3\left(2 i_{C}{ }^{s h}-i_{C}{ }^{s h 2}\right)+5\left(2 i_{C}{ }^{\text {bat }}-i_{C}^{\text {bat } 2}\right)}{8 l_{t}}\right]
\end{aligned}
$$

To obtain $i_{C}^{\text {sh }}, i_{C}^{\text {bat }}, l_{t}$ and $\varepsilon_{\text {ind }}$ one uses $\varepsilon_{\text {ind }} \equiv$ $R_{t} I_{C}^{\text {bat }} / i_{C}{ }^{\text {bat }}$ and Eqs. (14), (15) and (16b), with $Z_{\Omega}$ $I_{C}^{\text {res }}$ replaced by $R_{t} I_{C}^{\text {bat }} / i_{C}^{\text {bat }}$.

\section{CONCLUSIONS}

Measurements of plasma density during the first 5 orbits, and deorbiting rate over the remaining orbits, together with measurements of current at the cathodic end of the tether, can be used for a check of the OML current-collection law under a variety of conditions. Tests require an estimate of tether temperature (and consequently resistivity) at each point in orbit; a test failure might occasionally result from wrong estimates. Use of the battery mode in some primary cycles could obviate the need of a temperature estimate.

\section{ACKNOWLEDGMENTS}

The contributions of E.C. Lorenzini and M.L. Cosmo to this paper have been supported by NASA Marshall Space Flight Center through Grant NAG8-1605 with Leslie Curtis as Technical Monitor.

\section{REFERENCES}

1 Johnson, L., R.D. Estes, E.C. Lorenzini, M. MartínezSánchez and J.R. Sanmartín, J. Spacecraft and Rockets 37, 173 (2000). Lorenzini, E.C. et al., Annual report \#3, NASA Grant NAG8-1605 (July 2002).

3 Sanmartín, J.R. and R.D. Estes, Physics of Plasmas 6, 395 (1999) and 8, 4234 (2001); R.D. Estes and J.R. Sanmartín, Physics of Plasmas 7, 4320 (2000).

4 Sanmartín, J.R., M. Martínez-Sánchez and E. Ahedo, J. Propul. Power 9, 353 (1993). 